Urologe 2010 · 49[Suppl 1]:148

DOI 10.1007/s00120-010-2372-y

Online publiziert: 9. September 2010

(c) Springer-Verlag 2010

\author{
M.S. Michel \\ Urologische Klinik, Universitätsmedizin Mannheim
}

\title{
Akademie-Frühjahrsforum: Aktuelles \& Praxisrelevantes in der Urologie 2010
}

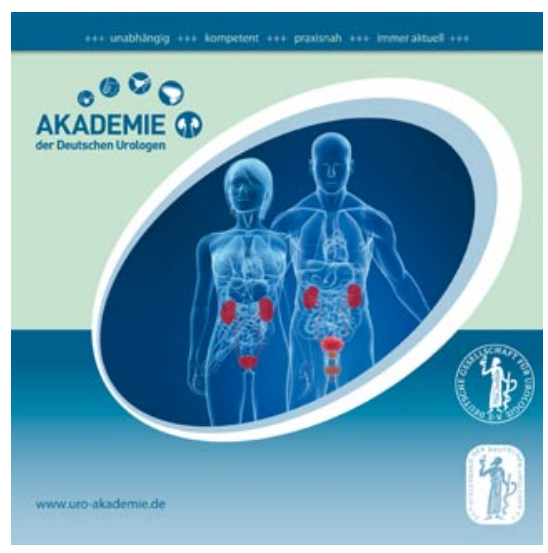

Im Rahmen der 51. Jahrestagung der Südwestdeutschen Gesellschaft für Urologie in Landau fand am 14.05.2010 erstmals das neu in das Programm der Akademie der Deutschen Urologen aufgenommene Akademie-Frühjahrsforum „Aktuelles \& Praxisrelevantes in der Urologie 2010" statt.

Beim Akademie-Frühjahresforum wurden die für die Urologie relevanten aktuellen Leitlinien und Publikationen themenbezogen dargestellt und kommentiert. Dabei wurde ein besonderes Augenmerk auf die Praxisrelevanz gelegt und den Teilnehmern Empfehlungen zur Umsetzung der gewonnen Erkenntnisse gegeben. Durch die Fokussierung auf das Wesentliche und die Aktualität war es möglich, in einem eintägigen Seminar diese Neuerungen darzustellen und dabei alle Gebiete der Urologie zu berücksichtigen. Hochrangige Referentinnen und Referenten bürgten für eine qualitativ hochwertige Fortbildung. Diese Veranstaltung war die am besten besuchte Veranstaltung der Tagung.

Das Akademie-Frühjahresforum stellt damit eine neue Facette des seitens der Akademie der Deutschen Urologen initiierten bundesweiten Fortbildungsangebotes dar, das anlässlich der Tagung der Südwestdeutschen Gesellschaft für Urologie in Landau unter Präsidentschaft von Prof. Molitor erstmalig angeboten wurde. Das Akademie-Frühjahrsforum war in den Kongress integriert und konnte ohne gesonderte Anmeldung besucht werden.
Das Akademie-Frühjahresforum wird keine einmalige Veranstaltung bleiben, sondern ist als wiederkehrende Veranstaltung ausgerichtet, die jährlich an verschiedenen Orten - vorzugsweise im Rahmen der Regionalkongresse - mit weitgehend gleich bleibenden Themen in gleich bleibend hoher Qualität, aber mit jeweils aktualisierten klinik- und praxisrelevanten Inhalten, angeboten wird. Nächstes Jahr findet das Akademie-Frühjahresforum auf mehreren Regionalkongressen statt und ermöglicht so einen flächendeckenden Zugang. Die Vorträge von 2010 sind in diesem Band zu Themenskriptforen gedruckt.

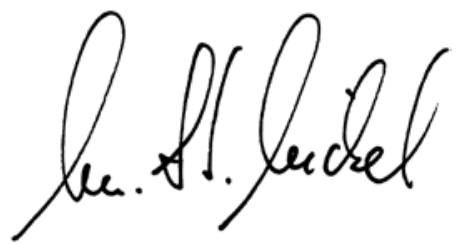

M.S. Michel

Korrespondenzadresse

Prof. Dr. M.S. Michel

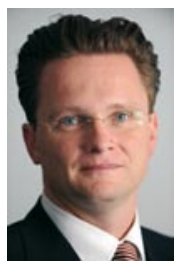

Urologische Klinik, Universitätsmedizin Mannheim Theodor-Kutzer-Ufer 1-3, 68135 Mannheim maurice-stephan. michel@umm.de 